\title{
Consecuencias alimentarias y nutricionales de la inseguridad alimentaria: la perspectiva de madres solteras
}

\author{
Nutritional consequences of the food \\ insecurity: the perspective \\ of single mothers
}

\begin{abstract}
Objective. To Investigate the consequences of food insecurity in adult women living in poverty in Mexico City. Subjects and Methods. During year 2007 ten focal groups with 44 single mothers who were beneficiaries of a governmental program in the Tlalpan County were carried out. Results. Testimonies of women indicate that food insecurity is related with overweight. This can be the result of a consumption of a monotonous diet based in cheap and high energy density foods, that they select food that they perceive produce satiety like bread, or that they experience cycles of restriction and binge eating, skip one or more meals and the coping with psychosocial stress. In some cases, food insecurity was related with loss of body mass. Conclusion. Food insecurity and urban poverty are related not only to nutritional deficiencies but also to obesity, one of the precursors of chronic diseases. Keys words: food insecurity, obesity, undernutrition, women, Mexico.
\end{abstract}

Elisabeth Hernández M. Diana Pérez $S$. Luis Ortiz-Hernández

Departamento de Atención a la Salud, Universidad Autónoma Metropolitana
unidad Xochimilco. México.
Dirigir la correspondencia:
Profesor
Luis Ortiz-Hernández
Universidad Autónoma Metropolitana unidad Xochimilco.
Calzada del Hueso 1100
Col. Villa Quietud
Coyoacán
México, 04960.
Tel.: (+52) (55) 54-83-72-43, Fax: (+52) (55) 54-83-72-18.
E-mail: lortiz@Correo.xoc.uam.mx.

Este trabajo fue recibido el 29 de Enero de 2013 y aceptado para ser publicado el 25 de Septiembre de 2013.

\section{INTRODUCCIÓN}

La inseguridad alimentaria ocurre cuando la disponibilidad o habilidad para adquirir los alimentos de maneras social y culturalmente aceptables son limitadas o inciertas (1, 2). La inseguridad alimentaria en los hogares está conformada por cuatro dimensiones (3): a) cuantitativa: no tener acceso a suficientes alimentos, b) cualitativa: no tener acceso a una dieta variedad y con calidad nutricional, c) Psicológica: ansiedad causada por la carencia de alimentos y la experiencia subjetiva del hambre. d) Social: obtener los alimentos de manera social y culturalmente aceptable. El principal determinante de la inseguridad alimentaria es la falta de recursos económicos, aunque otros aspectos también pueden condicionar su existencia (figura 1).

El año 2009, en México 23\% de los hogares con menores de edad experimentaban inseguridad leve, $16 \%$ inseguridad moderada y $17 \%$ inseguridad severa (4). En el Distrito Federal, durante el año 2003, el 42\% de la población se clasificaba en la categoría de seguridad alimentaria, 28\% inseguridad leve, $23 \%$ inseguridad moderada y el $7 \%$ sufría inseguridad severa; presentándose mayores niveles de inseguridad en los hogares en dónde existían niños que en aquellos que sólo eran habitados por adultos (5).
La inseguridad alimentaria compromete el consumo de alimentos, energía y nutrientes de las personas $(6,7)$. La experiencia de inseguridad alimentaria en los niños aumenta el riesgo de desnutrición, infecciones y distrés psicológico (8, 9); mientras que en las mujeres adultas se ha asociado con el riesgo de obesidad (10-12).

Los resultados de estos estudios cuantitativos pueden ser complementados con investigaciones cualitativas, con las cuales se conocería la perspectiva de las personas que sufren inseguridad alimentaria. Además, las investigaciones cualitativas permiten generar hipótesis de investigación y entender a profundidad los procesos bajo estudio (13). Por ejemplo, la relación entre obesidad e inseguridad alimentaria es, en primera instancia, contra intuitiva ya que la falta de acceso a los alimentos debería conducir a la pérdida de peso. Entre las explicaciones que se han postulado está el hecho de que las personas que sufren inseguridad alimentaria tienden a consumir alimentos económicos que en la mayoría de los casos tienen alta densidad energética (14). Sin embargo, otras explicaciones relacionadas con las conductas y las percepciones que tienen las personas respecto a la alimentación han sido insuficientemente documentadas.

La presente investigación se realizó con el objetivo de 
conocer las consecuencias alimentarias y nutricionales que tiene la inseguridad alimentaria en mujeres pobres que viven en el Distrito Federal.

\section{SUJETOS Y MÉTODOS}

En esta investigación se utilizó la metodología de los grupos focales lo que permite sondear sentimientos, opiniones, experiencias y percepciones de las personas (15). La obtención de la información se realizó como parte de las actividades de un proyecto de orientación alimentaria dirigido a mujeres que participaban en un programa gubernamental cuya población objetivo eran madres solteras pobres que tenían hijos inscritos en primaria o secundaria públicas. Las beneficiarias recibían una beca monetaria bimestral y capacitación para el autoempleo y el desarrollo de proyectos productivos. A las participantes se les solicitó su consentimiento informado para participar en el estudio y se obtuvo su autorización para realizar las grabaciones magnetofónicas garantizándoles confidencialidad. Se realizaron 10 grupos focales, en cada uno participaron de tres a seis mujeres. Al final se contaron con los testimonios de 44 mujeres. El proyecto fue aprobado por el Consejo Divisional de Ciencias Biológicas y de la Salud de la Universidad Autónoma Metropolitana, el cual sanciona tanto los aspectos científicos como los éticos.

Para la conducción de los grupos focales se siguieron las recomendaciones de Greenbaum (16). Cada grupo focal fue conducido por un moderador y un observador previamente capacitados quienes eran egresados de la Licenciatura en Nutrición. Los grupos se desarrollaron siguiendo una guía de tópicos previamente elaborada y consensada entre el equipo de investigación. Se buscó la mayor participación de todas las mujeres, sin presionar su intervención y buscando siempre crear un ambiente de confianza. El moderador tenía la consigna de abordar la guía de tópicos utilizando preguntas abiertas, profundizando temas relevantes para la investigación y respetando los términos utilizados por las participantes. Después de cada sesión, el observador daba retroalimentación al moderador del grupo respecto a su desempeño y a los temas cubiertos en la discusión.

Para analizar la información, las sesiones grupales fueron transcritas de manera textual usando el programa Microsoft Word. Usando el análisis de contenido $(17,18)$ se identificaron las categorías que emergieron en los diálogos de los grupos focales; realizando dos lecturas generales para identificar temas y palabras clave, a las cuales se les asignaron códigos. Esta lectura no se realizó para hacer inmediatamente un análisis del contenido, sino para tener un panorama general de los testimonios e identificar los temas emergentes, dejando abierta la posibilidad de que algunos sub-temas pudieran contenerse en ciertos temas. Durante una tercera lectura se codificaron los temas y códigos que previamente habían sido identificados. La codificación se realizó con el programa Ethnograph versión 6. Para garantizar la calidad del análisis de los datos (19), la primera autora (EHM) del estudio realizaba la codificación de las entrevistas, la cual posteriormente era presentada a los otros dos autores. Cuando existía una discrepancia en la codificación o la interpretación, el equipo de investigación discutía hasta llegar a un consenso.

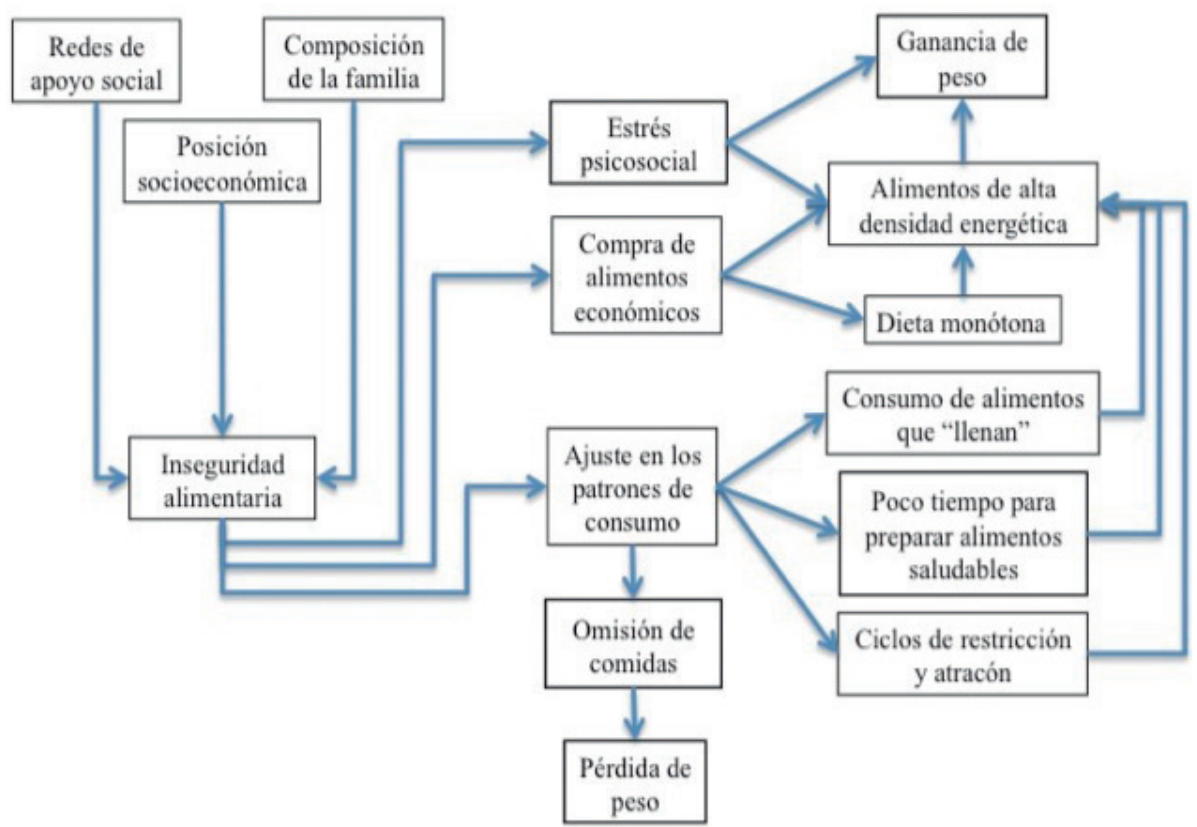

Las flechas que llegan a la inseguridad alimentaria son sus causas; mientras que las flechas que sale de la inseguridad son sus consecuencias. 


\section{RESULTADOS}

Una síntesis gráfica de los resultados se muestra en la figura 1. En los testimonios de las mujeres se constató que el precio de los alimentos define el tipo y la calidad de los alimentos que son adquiridos en el hogar $y$, consumen por sus miembros: al tiempo que dejan de comprar ciertos alimentos (frutas), adquieren aquellos que son de bajo costo (tortilla pan, sopa de pasta, arroz, frijoles y huevo). Con frecuencia, el resultado es una dieta monótona o poco variada. Estos hechos se pueden constatar con las siguientes verbalizaciones:

"¿qué es lo que compramos? pues para lo que nos alcanza y casi siempre es tortillas, pan y ... verdura, pero en pocas cantidades"

"Sí no tenemos dinero hacemos una olla de frijoles, mi mamá hace unas tostaditas con frijoles, nopalitos con frijoles" "ha habido ocasiones en las que no tenemos mucho dinero, entonces lo que hacemos es comprar un kilo de jitomate $y$ compro tortillas ... ipues no podemos comer de todo!"

"... cuando no tengo no compro fruta"

"eso es lo que van a comer, sopa o frijoles. Porque es para lo que me alcanza"

"entonces hay veces que o comen puro arroz blanco o compran una sopa así o hay veces que han llegado que una tortilla así frita con tantita sal"

La mayoría de las mujeres, por falta de recursos económicos, recurren al consumo de alimentos que, además de ser económicos, perciben que satisfacen el hambre. En ocasiones ellas son conscientes de que estos alimentos no tienen el mejor contenido nutrimental ni satisfacen sus necesidades y las de los miembros de la familia, como se verifica en los siguientes testimonios:

"Lo más fácil es tener muchos panes pues es lo más barato y eso lo utilizamos para llenarnos"

"Como no hay suficientes cosas, entonces ellos comen pan o tortillas y con lo que hay ya se llenan y ya quedan satisfechos con eso. Sé que eso no es una comida balanceada sino que se están llenando de pan y guisados nada más"

La reducción del número de comidas por parte de las mujeres también es una consecuencia de la inseguridad alimentaria que padecen estas familias:

"A veces desayuno, a veces no, a veces como o a veces no, ... porque a veces me alcanza el dinero"

"Hay ocasiones que nada más hago una comida al día, a veces dos"

Algunas mujeres expresaron que ante la falta de estabilidad en el ingreso, tanto ellas como los miembros de sus familias experimentan ciclos de restricción y "atracón" en su alimentación. Estas prácticas son reconocidas por los miembros de la familia como recurrentes y que deben aprender a acostumbrarse a ellas:

"Les digo, saben qué, tienen que comer cuando hay poquito de a poquito y cuando hay mucha pues mucha"

"Si tenemos en casa comida comemos lo suficiente y cuando no tenemos saben que no deben de pedir más"

Para asegurar el consumo de alimentos por sus hijos, las mujeres dejan de consumir una dieta variada y nutritiva, esto a pesar de tener el conocimiento de que los alimentos que dejan de consumir son saludables. De este modo, por falta de recursos económicos en ocasiones ellas recurren a la ingesta de alimentos que las "llenen" aunque éstos tengan bajo o escaso valor nutritivo:

"hago sopita y mínimo siempre procuro más a las niñas, aunque nosotros comamos huevo o frijoles, siempre les procuro su pollo, su carnita" "si tiene unos huevos, ... procura a los niños, aunque uno coma pura tortilla, pero no los deja uno sin comer" "Cuando seas mamá te vas a dar cuenta que prefieres que coman ellos, que tengan ellos completa su nutrición y tú ya te sacrificas a veces a no comer muchas cosas, no comprar cosas que también necesitas para tu nutrición o tu alimentación"

Algunas mujeres limitan su propia ingesta en un esfuerzo por amortiguar los efectos de la inseguridad alimentaria más severa en sus hijos.

"Muchas veces yo he dejado de comer más o dejar de comer

lo que es normal para cada quien por darles a ellos"

"Hace uno lo posible porque ellos no se queden sin comer aunque a veces uno se muere de hambre"

El recurrir al consumo de agua para mitigar el hambre fue comentado por las mujeres, quienes realizan esta práctica como otra manera de proteger la alimentación de sus hijos:

"Si me quedo con hambre pues lo que hago a veces es tomar agua, yo siento que con el agua se me pasa el hambre y siento que algo ya le cayo al estómago"

De acuerdo con las mujeres, la ganancia de peso, el tener sobrepeso o el no poder reducir el peso corporal son consecuencias que provoca la inseguridad alimentaria. Esto estuvo relacionado con factores como el tiempo prolongado que dedican al trabajo, la omisión de ciertas comidas y el ignorar la sensación de hambre en un momento para después sentirla en demasía:

"Luego no me da tiempo de comer y ya cuando llego ya a las 5 o 6 de la tarde tengo tanta hambre que me como todo lo que encuentro entonces, uno muchas veces trata de empezar el día ¿no? comiendo sano y poquito pero por lo mismo que no tengo tiempo para comer pues llegas $y$ cenas mucho, entonces al día siguiente dices: hay no, pues ya comí un montón, voy a desayunar un sándwich, ... y luego que no me gusta cargar mi bolsa ... con cosas como una fruta, entonces por eso no puedo bajar de peso"

"Desde que nació Pablo no tengo casi tiempo para mí, pues como lo que puedo y a la hora que pueda y ... a veces como a deshoras, a veces como en la noche porque no me da tiempo de comer en el horario que antes comía; yo me siento mal porque antes era muy delgada y ahora no lo soy $y$ he hecho esfuerzos para bajar de peso pero no puedo"

Las mujeres identificaron otras consecuencias por no comer, entre las que incluyen la gastritis y la pérdida de peso:

"En la tarde es cuando luego sí me mal paso por lo mismo de la escuela que no me da tiempo de comer y a consecuencia de eso pues tengo gastritis, porque si me he mal pasado"

"En muchas ocasiones yo si he bajado de peso por que si se me antoja cualquier cosa, no como"

En los testimonios existen manifestaciones de estrés psicosocial caracterizadas por resignación ("¿qué es lo que compramos? pues para lo que nos alcanza", "comemos lo que hay", "nos debemos de aguantar" o "y tú ya te sacrificas"), enojo ("ipues no podemos comer de todo!") y desesperación ("Hemos tenido situaciones difíciles" o "no me alcanza"). Parte de estas respuestas están relacionadas con su incapacidad para satisfacer las necesidades de sus hijos ("piden mucho... hemos tenido situaciones, si muy difícil, pero si son muchos gastos" o "Sé que eso no es una comida balanceada sino que se están llenando de pan y guisados nada más") o las de ellas mismas ("ya te sacrificas a veces a no comer muchas cosas, no comprar cosas que también necesitas para tu nutrición o tu alimentación"). Esas reacciones emocionales también pueden ser producto de su incapacidad para obtener lo que otras personas sí tienen ("Yo 
veo como la gente normal come frutas, carne, verduras y pues nosotros comemos frijoles, sopa y compro tortillas", "le digo que no comemos las tres comidas" o "yo he dejado de comer ... lo que es normal para cada quien"). Finalmente, es posible que el estrés psicosocial se deba a que saben que sus hijos son conscientes de las dificultades que existen en la familia para acceder a la comida y ellas no quieren que eso ocurra:

"Pues yo a veces si me he quedado sin comer porque les estoy dejando para el desayuno, aunque luego me dicen "ya comiste" y trato de eludir, para que no dejen de comer ellos"

\section{DISCUSIÓN}

Los resultados de este estudio coinciden con la teoría (20) y la evidencia empírica disponible (1), que las familias con inseguridad alimentaria limitan la variedad de alimentos en función de los recursos con los que cuentan. Una primera consecuencia es que dejan de adquirir y consumir los alimentos de mayor costo, algunos de los cuales son saludables como las frutas, verduras, lácteos y carnes $(7,21)$. Algunas mujeres de la ciudad de México expresaron que cuando su ingreso se los permitía compraban frutas de temporada, porque son baratas; sin embargo, la mayoría expresó que dan prioridad a sus hijos para que las consuman. De este modo, las mujeres-como jefas de familia- con frecuencia sólo pueden consumir los mismos alimentos que son económicos, lo que resulta en una dieta limitada y monótona. Esta dieta monótona se compone por tortilla, sopa de pasta, arroz, pan, frijol y huevo; los cuales en México son los alimentos de menor precio (22).

En los testimonios se hizo evidente que la adquisición y consumo de ciertos alimentos no sólo están dados por razones económicas; también existen motivos relacionados con la experiencia corporal asociada al consumo de los mismos. Para las mujeres es relevante la percepción de la capacidad de los alimentos para generar saciedad. La tortilla, la sopa de pasta y el pan son alimentos consumidos cotidianamente porque su consumo reduce la sensación de hambre y produce saciedad, además de ser económicos.

Otro hallazgo de este estudio fue que tanto la obesidad como la pérdida de peso son problemas de nutrición asociados con la inseguridad alimentaria. La evidencia epidemiológica $(23,24)$ muestra que el mayor riesgo de obesidad se presenta en la inseguridad alimentaria leve o moderada, mientras que la inseguridad severa se puede relacionar con pérdida de peso. Esta relación curvilínea entre inseguridad alimentaria y riesgo de obesidad puede ser explicada (14, 23, 24): cuando en los hogares la inseguridad es leve, las mujeres tienden a ganar peso porque su consumo se basa principalmente en alimentos de bajo costo y alta densidad energética; mientras que cuando la inseguridad es severa los miembros de la familia dejan de comer y es cuando existe pérdida de peso.

Los resultados de este estudio han evidenciado algunos posibles mecanismos que podrían explicar la relación de la inseguridad alimentaria con la obesidad en mujeres adultas. Dos ya han sido mencionados: en los hogares con inseguridad se adquieren alimentos que son económicos y de alta densidad energética; además, se busca consumir alimentos que se percibe generan saciedad. Otra explicación podrían ser los ciclos de restricción y atracón que experimentan las personas que tienen ingresos inestables. Este patrón de consumo es verbalmente reforzado por las madres a sus hijos, con lo cual puede ser percibido como necesario o "natural". Vinculado a lo anterior, es que parte de la experiencia de la inseguridad alimentaria puede implicar el omitir una o varias comidas al día o, incluso, dejar de comer todo el día. Al respecto, existe evidencia que el reducir el número de comidas (25), omitir el desayuno (26) o los ciclos de restricción dietética (27) se asocian con ganancia de peso y / u obesidad.

Nuestros hallazgos pueden indicar que la experiencia de la inseguridad alimentaria y sus efectos en el peso de las mujeres están relacionados con su papel como madres. Se ha reportado que en mujeres adultas casadas, viudas y divorciadas la relación entre inseguridad alimentaria y obesidad fue positiva; pero en las solteras tal asociación no existió (11) o la asociación fue negativa (10). Las madres, en su afán de proteger la ingesta de sus hijos, deciden consumir una dieta poco variada, esto a pesar de que saben que no es saludable $(2,28)$. La protección del consumo de alimentos de los niños no sólo se da al limitar su propio consumo. Las mujeres también deben trabajar largas jornadas, lo cual les dificulta realizar un número adecuado de comidas, además de no contar con tiempo suficiente para preparar y consumir alimentos saludables.

Socialmente se espera que las madres cubran las necesidades de sus hijos y el hecho de experimentar inseguridad puede implicar que no cumplen con esa función, lo cual puede experimentarse como un estresor. Es posible que las mujeres experimenten estrés pues son conscientes de que no tienen acceso a satisfactores que otras personas sí tienen, además de que pueden estar preocupadas que sus propios hijos sufran por ser conscientes de esa situación. El estrés psicosocial se ha relacionado con el desarrollo de obesidad (29). Otros malestares mencionados por las mujeres como úlceras gástricas y gastritis también podrían estar relacionados con el estrés derivado de la inseguridad alimentaria.

Una de las limitaciones del presente estudio es que no se incluyeron a mujeres que vivieran con su pareja, con lo cual se limita la posibilidad de transferir los hallazgos a otros grupos. Además, los resultados del presente estudio no pueden ser considerados como evidencia para establecer la existencia de relaciones causales. Sin embargo, los testimonios de las mujeres permiten generar hipótesis que en el futuro podrían se evaluadas con estudios diseñados ex profeso. Otra limitación se debe a que el estudio fue realizado hace siete años. Sin embargo, desde entonces pocos cambios han existido en las condiciones que generan la inseguridad alimentaria (i.e. pobreza y desigualdad socioeconómica), así como en los contextos en los que se ha dado el incremento de la obesidad en la región. Por ello, es previsible que las situaciones descritas por las mujeres que entrevistamos sigan siendo vigentes. Muestra de ello son la reciente publicación sobre el tema hecha por investigadores brasileños (12).

Este estudio muestra que las poblaciones empobrecidas pueden ser especialmente vulnerables al efecto del ambiente obesigénico (alta disponibilidad de alimentos densos en energía). Por ello, las políticas y los programas que estén encaminados a promover estilos de vida saludables entre las personas en situación de pobreza necesariamente tiene que contemplar como objetivo el lograr que los alimentos saludables sean accesibles para ellas. Aunque los programas de transferencia monetaria tienen un efecto en la reducción de la pobreza, es mínimo por lo cual conviene considerarlos como parte de una política social y económica que realmente logre la erradicación de la inseguridad alimentaria. La regulación efectiva de las condiciones de empleo y de los salarios son medidas gubernamentales que pueden tener impacto en la reducción de la inseguridad alimentaria en los hogares. De igual modo, los subsidios focalizados a alimentos saludables 
pueden ser medidas que promuevan el consumo de estos alimentos en las familias de bajos ingresos. Finalmente, los programas gubernamentales de asistencia alimentaria y de educación nutricional deben ser ajustados para reconocer que la principal razón por la que los hogares de bajos ingresos no consumen alimentos saludables es por que no pueden adquirirlos; mientras que la falta de conocimientos y la voluntad para hacerlo tienen un papel marginal.

\section{RESUMEN}

Objetivo. Conocer las consecuencias alimentarias y nutricionales de la inseguridad alimentaria en mujeres adultas en condiciones de alta marginalidad de la Ciudad de México. Sujetos y Métodos. Durante el año 2007 se efectuaron diez grupos focales con 44 madres solteras pobres, beneficiarias de un programa gubernamental en la delegación Tlalpan, Distrito Federal. Resultados. Los testimonios de las mujeres indican que la inseguridad alimentaria se relaciona con el incremento de peso corporal. Esto puede ser atribuido a que consumen una dieta monótona basada en alimentos que son económicos y de alta densidad energética, buscan consumir alimentos que perciben generan saciedad como los cereales, experimentan ciclos de restricción y atracón, omiten una o varias comidas al día y experimentan estrés psicosocial. En algunos casos, la inseguridad alimentaria también se relacionó con pérdida de peso. Conclusiones. La experiencia de la inseguridad alimentaria y la pobreza urbana no sólo se relacionan con carencias nutricionales sino también con uno de los precursores de enfermedades crónicas: la obesidad.

Palabras clave: Inseguridad alimentaria, obesidad, desnutrición, mujeres, México.

Agradecimientos: Los autores agradecen a las mujeres que participaron en el estudio por compartirnos sus experiencias y enseñarnos cómo lidian con la adversidad. También reconocemos el trabajo de Mabel Meza, Coral Martínez y Juan Martínez, cuya participación en la conducción de los grupos focales fue esencial. También agradecemos el trabajo de Norma Fuentes, Jorge Ávila y Mario Flores, quienes desarrollaron las actividades de orientación alimentaria para las mujeres participantes en el estudio.

\section{BIBLIOGRAFÍA}

1. Kaiser LL, Melgar-Quiñonez H, Townsend MS, Nicholson $Y$, Fujii ML, Martin AC, et al. Food insecurity and food supplies in Latino households with young children. J Nutr Educ Behav 2003; 35: 148-53.

2. Comité Científico de la ELCSA. Escala Latinoamericana de Seguridad Alimentaria (ELCSA): manual de uso y aplicaciones. Santiago de Chile: Organización de las Naciones Unidas para la Agricultura y la Alimentación 2012.

3. Radimer KL, Olson CM, Campbell CC. Development of indicators to assess hunger. J Nutr. 1990; 120 (Supp/ 11): 1544-8.

4. Consejo Nacional de Evaluación de la Política de Desarrollo Social, Fondo de Naciones Unuidas para la Infancia. La niñez y la adolescencia en el contexto de la crisis económica global: el caso de México. México: Consejo Nacional de Evaluación de la Política de Desarrollo Social, Fondo de Naciones Unidas para la Infancia, 2010.

5. Parrás PPR. Inseguridad alimentaria en México. Reforma. 29 de junio, 2008.

6. Leyna GH, Mmbaga EJ, Mnyika KS, Hussain A, Klepp $K I$. Food insecurity is associated with food consumption patterns and anthropometric measures but not serum micronutrient levels in adults in rural Tanzania. Public Health Nutr. 2010; 13(9): 1438-44.

7. Ortiz-Hernández L, Acosta-Gutiérrez MN, Núñez-Pérez $A E$, Peralta-Fonseca N, Ruiz-Gómez Y. En escolares de la Ciudad de México la inseguridad alimentaria se asoció positivamente con el sobrepeso. Rev Invest Clin. 2007; 59(1): 32-41.

8. Hackett M, Melgar-Quiñonez H, Alvarez MC. Household food insecurity associated with stunting and underweight among preschool children in Antioquia, Colombia. Rev Panam Salud Pública 2009; 25(6): 506-10.

9. Kleinman RE, Murphy JM, Little $M$, Pagano $M$, Wehler $C A$, Regal $K$, et al. Hunger in children in the United States: potential behavioral and emotional correlates. Pediatrics 1998; 101(1): E3.

10. Ortiz-Hernández L, Barrera MJ, Blanco E. Asociación de la inseguridad alimentaria con el sobrepeso y la obesidad en mujeres de la ciudad de México. Nutr Clin. 2007; 10(2): 63-70.

11. Hanson KL, Sobal J, Frongillo EA. Gender and marital status clarify associations between food insecurity and body weight. J Nutr. 2007; 137(6): 1460-5.

12. Velasquez-Melendez G, Schlussel MM, Brito AS, Silva $A A$, Lopes-Filho JD, Kac G. Mild but not light or severe food insecurity is associated with obesity among Brazilian women. J Nutr. 2011; 141(5): 898-902.

13. Muntaner C, Gomez MB. Qualitative and quantitative research in social epidemiology: is complementarity the only issue? Gac Sanit 2003; 17 (Suppl 3): 53-7.

14. Ortiz-Hernández L, Rivera JA. Inseguridad alimentaria y riesgo obesidad. En: García-García E, Kaufer-Hortwitz. Pardía J, Arroyo P (Editores). La obesidad. Perspectivas para su comprensión y tratamiento. México, INCMNSZ, FUNSALUD, Editorial Médica Panamericana, 2010: pp 35-42.

15. Pérez C. Sobre la metodología cualitativa. Rev Esp Salud Pública 2002; 76(5): 373-80.

16. Greenbaum TL. Moderating focus groups. A practical guide for group facilitation. Thousand Oaks: Sage Publications; 2000.

17. Coffey A, Atkinson P. Los conceptos y la codificación. Encontrar el sentido a los datos cualitativos. Estrategia complementarias de investigación. Universidad de Antioquia, 2003: 31-63.

18. Pope C, Ziebland S, Mays N. Qualitative research in health care. Analyzing qualitative data. Br Med J. 2000; 320(7227): 114-6.

19. Mays N, Pope C. Qualitative research in health care. Assessing quality in qualitative research. Br Med J. 2000; 320(7226): 50-2.

20. Bickel GN, M.; Price, C.; Hamilton, W.; Cook, J. Guide to measuring household food security. Revised 2000. Alexandria VA: U.S. Department of Agriculture, Food Nutr Service, 2000

21. Kendall A, Olson CM, Frongillo EA, Jr. Relationship of hunger and food insecurity to food availability and consumption. J Am Diet Assoc. 1996; 96(10): 1019-24.

22. Ortiz-Hernández L. Evolución de los precios de los alimentos y nutrimentos en México entre 1973 y 2004 Arch Latinoam Nutr. 2006;56(3):201-15.

23. Adams EJ, Grummer-Strawn L, Chavez G. Food insecurity is associated with increased risk of obesity in California women. J Nutr. 2003;133(4):1070-4.

24. Townsend MS, Peerson J, Love B, Achterberg C, Murphy 
SP. Food insecurity is positively related to overweight in women. J Nutr. 2001; 131(6): 1738-45.

25. Chapelot D, Marmonier C, Aubert R, Allegre C, Gausseres $N$, Fantino $M$, et al. Consequence of omitting or adding a meal in man on body composition, food intake, and metabolism. Obes. 2006; 14(2): 215-27.

26. Cho S, Dietrich M, Brown CJ, Clark CA, Block G. The effect of breakfast type on total daily energy intake and body mass index: results from the Third National Health and Nutrition Examination Survey (NHANES III). J Am Coll
Nutr. 2003; 22(4): 296-302.

27. Pietilainen $K H$, Saarni SE, Kaprio J, Rissanen A. Does dieting make you fat? A twin study. Int J Obes. 2012; 36(3): 456-64.

28. Melgar-Quiñonez H, Kaiser LL, Martin AC, Metz D, Olivares $A$. Inseguridad alimentaria en latinos de California: observaciones de grupos focales. Salud Pública Mex 2003; 45(3): 198-205.

29. Bjorntorp P. Do stress reactions cause abdominal obesity and comorbidities? Obes Rev. 2001; 2(2): 73-86. 